\title{
Early durotomy with duroplasty for severe adult spinal cord injury without radiographic abnormality: a novel concept and method of surgical decompression
}

\author{
Fengzhao Zhu ${ }^{1}$. Sheng Yao ${ }^{1}$. Zhengwei Ren ${ }^{1}$ - Dionne Telemacque ${ }^{1}$ - Yanzhen $\mathrm{Qu}^{1} \cdot$ Kaifang Chen $^{1} \cdot$ Fan Yang $^{1}$. \\ Lian Zeng ${ }^{1} \cdot$ Xiaodong Guo ${ }^{1}$ (i)
}

Received: 14 June 2019 / Revised: 25 July 2019 / Accepted: 31 July 2019 / Published online: 22 August 2019

(c) The Author(s) 2019

\begin{abstract}
Purpose Treatment options for adult spinal cord injury without radiographic abnormality (ASCIWORA) varied. Compression of ASCIWORA may more likely result from spinal cord lesions such as edema and hemorrhage or contusion. This study aimed to explore the clinical effect of early durotomy with duroplasty decompression in the treatment of severe ASCIWORA. Methods Data of 16 patients with ASCIWORA who underwent early $(<72 \mathrm{~h})$ posterior laminectomy followed by durotomy with duroplasty decompression from June 2015 to January 2017 were retrospectively analyzed. Patients' prognosis was analyzed by American Spinal Injury Association Impairment Scale (AIS) grades and scores. In 3 patients, intraspinal pressure (ISP) was continuously monitored for 1 week.

Results Cervical magnetic resonance imaging (MRI) revealed spinal cord edema in 9 patients and suspected hemorrhage or contusion in 7 cases. Pathological manifestations of spinal cord injury found during the operation were consistent with preoperative MRI findings. Of the 16 cases, AIS grade was improved by 1 grade in 3 cases, 2 grades in 11 cases, and 3 grades in 1 case. The AIS scores at the last follow-up were significantly higher than preoperative scores. There was a high level of ISP after laminectomy, whereas ISP continued to decrease steadily after durotomy.

Conclusions Durotomy helps thoroughly decompress the spinal cord and improve cerebrospinal fluid circulation in severe ASCIWORA cases. Cervical MRI and pathological investigation of the spinal cord can be used to evaluate and predict the prognosis of ASCIWORA patients. ISP monitoring is an effective method for evaluating intramedullary pressure and decompression.
\end{abstract}

\section{Graphic abstract}

These slides can be retrieved under Electronic Supplementary Material.
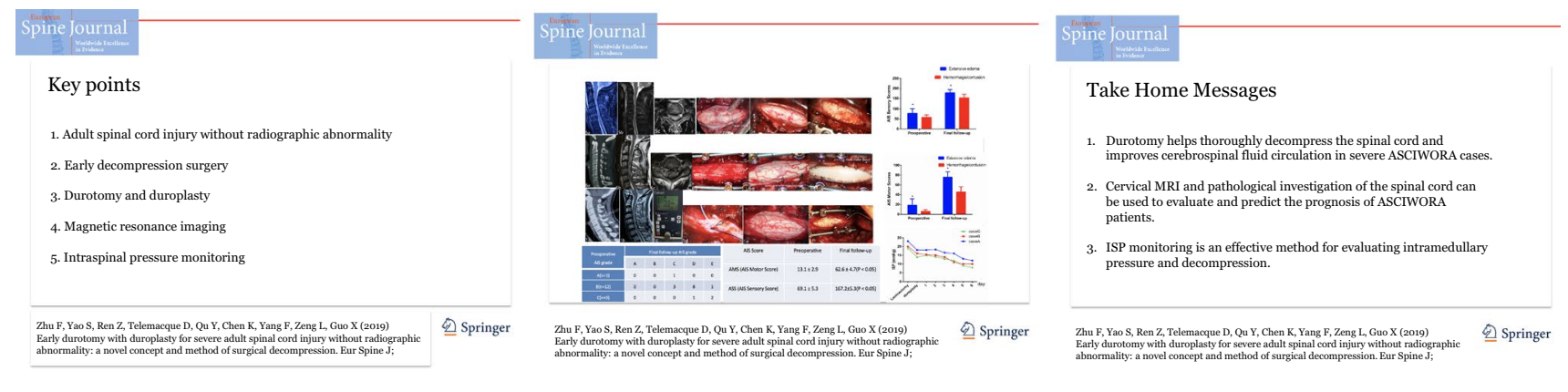

Fengzhao Zhu and Sheng Yao have contributed equally to this paper.

Electronic supplementary material The online version of this article (https://doi.org/10.1007/s00586-019-06091-1) contains supplementary material, which is available to authorized users.

Extended author information available on the last page of the article 
Keywords Adult spinal cord injury without radiographic abnormality · Early decompression surgery · Durotomy and duroplasty $\cdot$ Magnetic resonance imaging $\cdot$ Intraspinal pressure monitoring

\section{Introduction}

Spinal cord injury without radiographic abnormality (SCIWORA) was first described by Pang and Wilberger in paralyzed children [1]. Previous opinions have suggested that SCIWORA rarely occurs in adults. Recently, clinical studies have reported that the incidence of SCIWORA in adults is very high and that the actual rate is underestimated [2]. Unlike the injury mechanism in children, SCIWORA in adults is often accompanied by cervical degeneration, which can lead to spinal cord injury (SCI) under mild trauma such as a hyperextension injury [3]. To distinguish it from pediatric cases, we suggest that this disease be called ASCIWORA. In the past, more discussions focused on the comparison of surgical and conservative treatment and the surgical timing for ASCIWORA. The management of spinal cord lesion, such as spinal cord edema and hemorrhage or contusion, has received little attention. However, such SCI largely results from a spinal cord lesion itself. Severe spinal cord edema or hemorrhage can lead to a significant increase in intraspinal pressure (ISP), while the continuous increase in ISP would lead to a vicious cycle of "ischemia-edema-ischemia" and result in secondary injury [4]. Phang et al. measured the ISP and concluded that laminectomy decompression is not enough to reduce ISP after acute SCI, and laminectomy with durotomy can achieve complete decompression [5]. We tried early durotomy with duroplasty in SCI with fracture and dislocation and achieved some meaningful clinical results [6]. There is no significant osseous compression in ASCIWORA, and the spinal meninges compression plays a more important role. Therefore, we adopted durotomy with duroplasty decompression to treat these diseases from June 2015 to January 2017. We aimed to explore the clinical effect of early durotomy with duroplasty decompression in the treatment of severe ASCIWORA.

\section{Methods}

\section{Patients}

This study retrospectively analyzed data of 16 patients who underwent durotomy with duroplasty decompression for ASCIWORA from June 2015 to January 2017. The Ethics Committee of the Hospital and Medical College approved this study and waived the requirement for informed consent. The inclusion criteria were as follows: age $>18$ years, AIS grade for severe ASCIWORA was C and below preoperatively, no fracture or dislocation was observed in $\mathrm{X}$-ray and computed tomography (CT) of the cervical spine, and cervical magnetic resonance imaging (MRI) showed spinal cord edema, hemorrhage, or contusion on T2 images. The exclusion criteria were as follows: preoperative AIS grade was D and E, fractures or dislocations were observed on X-ray and CT of the cervical spine, and cervical MRI showed almost normal $\mathrm{T} 2$ images. All patients were admitted to our hospital within $48 \mathrm{~h}$ after injury.

\section{Preoperative physical examination and imaging examination}

Physical examination was performed immediately after admission to our hospital. AIS grade and scores of all patients were recorded. Emergency cervical X-ray imaging was performed immediately after patient assessment; some cases were examined directly by cervical CT and MRI. In our hospital, the multidisciplinary treatment team for SCI can complete MRI examination within $2 \mathrm{~h}$.

\section{Surgical procedure}

All 16 patients hospitalized within $48 \mathrm{~h}$ completed the surgery within $72 \mathrm{~h}$. We adopted the same surgical procedure: posterior laminectomy followed by durotomy with duroplasty. After an extensive laminectomy, the spinal cord pulsation (CSF circulation) did not recover in all cases. Then, bilateral cervical lateral mass screw and rods (Sofamor/Danek pedicle screw-rod system) were inserted. The extent of laminectomy and durotomy was determined based on the segment with spinal cord edema. Under the microscope, the dura was cut longitudinally, pulled up using a micro-nerve hook, and dissected slowly longitudinally. When the incision was wide enough, the silk thread pulled both sides of the dura incision. The hematoma on the surface of the spinal cord was removed thoroughly. After repeated rinsing with physiological saline, the dura was expanded and sutured with artificial dura mater patch (Aesculap AG Neuro-Patch). We opened a window of about $2-4 \mathrm{~mm}$ on the surface of the dura and placed the Codman probe into the dura mater to measure the ISP after laminectomy (Fig. 1). The values were recorded after the reading remained unchanged. ISP was measured and recorded again after durotomy with duroplasty. The probe was placed subdurally for 1 week, and the subdural pressure was recorded every $2 \mathrm{~h}$. Posterior fusion was performed in all cases. 


\section{Statistical analysis}

Data were analyzed using the SPSS statistical software (version 20.0; SPSS, Inc., Chicago, IL, USA). Paired $t$-test was used for the preoperative and final follow-up comparison. $P$ value $<0.05$ was considered statistically significant.

\section{Results}

This study included 16 patients ( 13 men and 3 women), aged 22-65 (average age, 47.5) years. Among them, 8 cases were caused by car accidents, 7 cases were caused by falling, and 1 case was caused by heavy damage to the neck. Preoperative physical examination according to AIS grade was as follows: 1 case of AIS grade A, 12 cases of AIS grade B, and 3 cases of AIS grade C. Of the 16 cases, $14(87.5 \%)$ had degenerative changes of the cervical spine. The cervical T2 MRI showed spinal cord edema in 9 cases and spinal cord hemorrhage or contusion in 7 cases.

All surgeries were successfully completed. The surgery time was $120-200 \mathrm{~min}$, and the average time was $155 \mathrm{~min}$. The bleeding volume was $280-500 \mathrm{ml}$, and the average bleeding volume was $350 \mathrm{ml}$. X-ray, CT, and MRI results of the cervical spine were reviewed after operation. The followup time was 9-28 months, with an average of 18 months. All patients achieved cervical fusion; the fusion time range was 3.5-6 months, and the average fusion time was 4.5 months.

Detailed physical examinations were performed at the last follow-up, and AIS motor scores (AMS) and AIS sensory scores (ASS) of all 16 cases were recorded (Table 1). We found that AIS grade was improved by 1 grade in 4 cases, 2 grades in 11 cases, and 3 grades in 1 case (Table 2). The AMS and ASS at the last follow-up were significantly higher than those before operation, and there were significant differences between the AMS and ASS (Table 3).
Among 16 cases, we divided the patients into two groups according to their manifestations. Group 1 had 7 cases of spinal cord hemorrhage or contusion, and group 2 had 9 cases of single spinal cord edema. The preoperative AMS and ASS of group 2 were higher than those of group 1 (Table 4). The AMS and ASS of group 2 were also higher than those of group 1 at the last follow-up (Table 5), indicating that single spinal cord edema is less severe than contusion or hemorrhage and has a better prognosis (Fig. 2).

ISP monitoring was performed in 3 patients within $72 \mathrm{~h}$ of injury, whose ISP after laminectomy was high. ISP was measured again, and the values were recorded after durotomy with duroplasty (Table 6). The ISP decreased steadily during the succeeding week of continuous monitoring (Fig. 3), and no neurological deterioration occurred during ISP monitoring.

Three patients had CSF leakage and underwent lumbar cistern drainage. The cervical drainage tube was removed within 3 days after the operation. The drainage tube of the lumbar cistern was removed in 10 days. All incisions healed within 3 weeks. Postoperative neck pain occurred in 2 patients. All patients were treated in the rehabilitation department for 3-4 months after surgery.

\section{Discussion}

The results of this study show that the overall prognosis of patients with spinal cord edema is better than that in patients with hemorrhage or contusion. Surprisingly, patients with spinal cord hemorrhage or contusion who were preoperatively graded as AIS grade A and AIS grade B achieved some recovery after surgery. This suggests that not all patients with spinal cord hemorrhage or contusion have a poor prognosis. Early and adequate decompression may contribute to the recovery of neurological function. Thus, there is an urgent need of a large number of clinical cases in prospective clinical studies to confirm its effectiveness. A

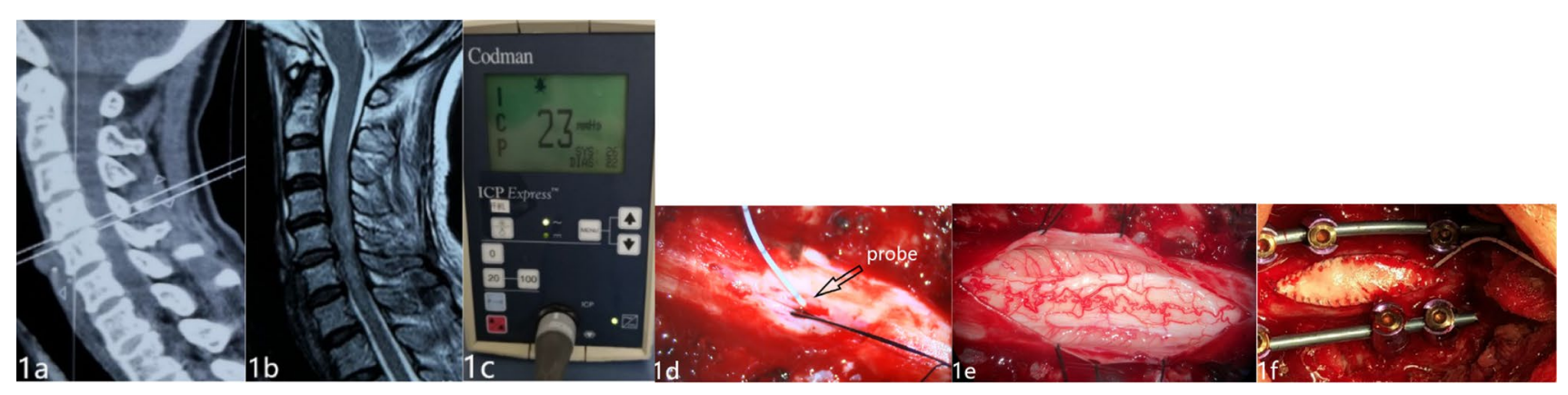

Fig. 1 Surgery was done in $72 \mathrm{~h}$. a, b A case with ASCIWORA. c The Codman manometer used in this study. d After laminectomy, we placed the probe subdurally. e Alleviation of the compression of the spinal cord and spinal cord expansion after durotomy. $\mathbf{f}$ The probe was placed subdurally after duroplasty. The AIS grade increased from B to C 
Table 1 Clinical summary of patients with ASCIWORA

\begin{tabular}{lllllll}
\hline Cases & Sex/age & Injury mechanism & Degeneration & $\begin{array}{l}\text { Operation } \\
\text { time }(\mathrm{h})\end{array}$ & MRI & AIS grade \\
\hline 1 & M/49y & Falling & Yes & $<48$ & Extensive edema & $\mathrm{B} \rightarrow \mathrm{D}$ \\
2 & M/65y & Falling & Yes & $<24$ & Extensive edema & $\mathrm{C} \rightarrow \mathrm{D}$ \\
3 & F/44y & Car accident & Yes & $<24$ & Extensive edema & $\mathrm{B} \rightarrow \mathrm{D}$ \\
4 & M/49y & Falling & Yes & $<24$ & Extensive edema & $\mathrm{B} \rightarrow \mathrm{D}$ \\
5 & M/44y & Car accident & Yes & $<24$ & Extensive edema & $\mathrm{B} \rightarrow \mathrm{D}$ \\
6 & M/48y & Car accident & Yes & $<24$ & Hemorrhage/contusion & $\mathrm{B} \rightarrow \mathrm{C}$ \\
7 & M/51y & Heavy attack & No & $<24$ & Hemorrhage/contusion & $\mathrm{A} \rightarrow \mathrm{C}$ \\
8 & M/55y & Falling & Yes & $<24$ & Hemorrhage/contusion & $\mathrm{B} \rightarrow \mathrm{D}$ \\
9 & M/45y & Falling & Yes & $<72$ & Extensive edema & $\mathrm{C} \rightarrow \mathrm{E}$ \\
10 & M/52y & Falling & Yes & $<24$ & Extensive edema & $\mathrm{C} \rightarrow \mathrm{E}$ \\
11 & M/42y & Falling & Yes & $<48$ & Extensive edema & $\mathrm{B} \rightarrow \mathrm{E}$ \\
12 & M/56y & Car accident & Yes & $<48$ & Extensive edema & $\mathrm{B} \rightarrow \mathrm{D}$ \\
13 & F/42y & Car accident & Yes & $<72$ & Hemorrhage/contusion & $\mathrm{B} \rightarrow \mathrm{C}$ \\
14 & M/22y & Car accident & No & $<72$ & Hemorrhage/contusion & $\mathrm{B} \rightarrow \mathrm{D}$ \\
15 & F/46y & Car accident & Yes & $<72$ & Hemorrhage/contusion & $\mathrm{B} \rightarrow \mathrm{D}$ \\
16 & M/50y & Car accident & Yes & $<24$ & Hemorrhage/contusion & $\mathrm{B} \rightarrow \mathrm{C}$ \\
\hline
\end{tabular}

Table 2 Preoperative and final follow-up AIS grade of our series

\begin{tabular}{llllll}
\hline Preoperative AIS grade & \multicolumn{5}{l}{ Final follow-up AIS grade } \\
\cline { 2 - 6 } & A & B & C & D & E \\
\hline A $(n=1)$ & 0 & 0 & 1 & 0 & 0 \\
B $(n=12)$ & 0 & 0 & 3 & 8 & 1 \\
C $(n=3)$ & 0 & 0 & 0 & 1 & 2 \\
\hline
\end{tabular}

Table 3 Preoperative and final follow-up AIS scores in this series

\begin{tabular}{lcr}
\hline AIS score & Preoperative & \multicolumn{1}{c}{ Final follow-up } \\
\hline AMS (AIS motor score) & $13.1 \pm 2.9$ & $62.6 \pm 4.7(P<0.05)$ \\
ASS (AIS sensory score) & $69.1 \pm 5.3$ & $167.2 \pm 5.3(P<0.05)$ \\
\hline
\end{tabular}

Table 4 Preoperative AIS scores between group 1 and group 2

\begin{tabular}{lcr}
\hline Preoperative & Edema & \multicolumn{1}{c}{ Hemorrhage/contusion } \\
\hline AMS (AIS Motor Score) & $18.6 \pm 4.3$ & $6.1 \pm 1.2(P=0.0439<0.05)$ \\
ASS (AIS Sensory Score) & $78.0 \pm 7.6$ & $57.6 \pm 4.8(P=0.0472<0.05)$ \\
\hline
\end{tabular}

Table 5 Final follow-up AIS scores between group 1 and group 2

\begin{tabular}{lrl}
\hline Final follow-up & \multicolumn{1}{l}{ Edema } & Hemorrhage/contusion \\
\hline $\begin{array}{l}\text { AMS (AIS Motor } \\
\text { Score) }\end{array}$ & $75.9 \pm 3.6$ & $45.6 \pm 4.0(P=0.0021<0.05)$ \\
$\begin{array}{l}\text { ASS (AIS Sensory } \\
\text { Score) }\end{array}$ & $178.4 \pm 5.3$ & $153.1 \pm 6.8(P=0.0343<0.05)$ \\
\hline
\end{tabular}

thorough understanding of the pathology of human SCI is helpful in the selection of treatment and judgment of prognosis [7].

\section{Selection of surgical methods}

The choice of surgical methods is varied. Despite the controversies, most doctors agree that adequate decompression is the main criterion for the choice of surgical methods regardless of the type of surgery [8]. Bony decompression has received widespread attention, while decompression of the spinal cord itself has rarely been mentioned. The injury mechanism of ASCIWORA is different from that in patients with fracture and dislocation. Bony compression of the spinal cord is very severe in patients with fracture and dislocation. For ASCIWORA, endogenous lesions of the spinal cord may have a greater influence.

As early as 1905, Allen performed spinal durotomy and myelotomy in mice with SCI [9]. Phang concluded that laminectomy decompression alone could not effectively reduce the ISP. Spinal durotomy and duroplasty could reduce the ISP and increase the spinal cord perfusion pressure (SCCP) by measuring the ISP of SCI patients [5]. Saadoun found that lower ISP and higher SCCP were thought to contribute to the recovery of neurological function by measuring changes in ISP and assessing the AIS grade [10]. Feng pointed out that spinal cord decompression may contribute to the neurological recovery in additional to bony decompression [11].

The dura was considered tough and inelastic and may significantly affect the biomechanical properties of human SCI [12]. Therefore, when spinal cord edema or hemorrhage occurs in SCI, the dura significantly limits the expansion of 

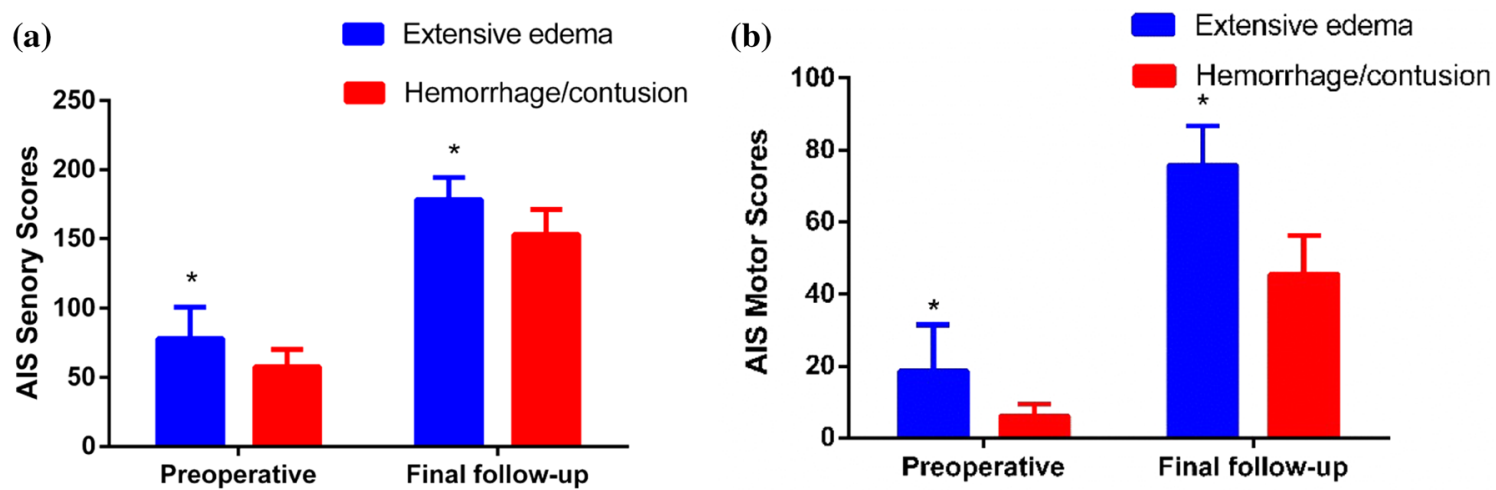

Fig. 2 Comparison of a ASS and b AMS at preoperative and final follow-up. AMS American Spinal Injury Association Impairment Scale motor scores, ASS American Spinal Injury Association Impairment Scale sensory scores

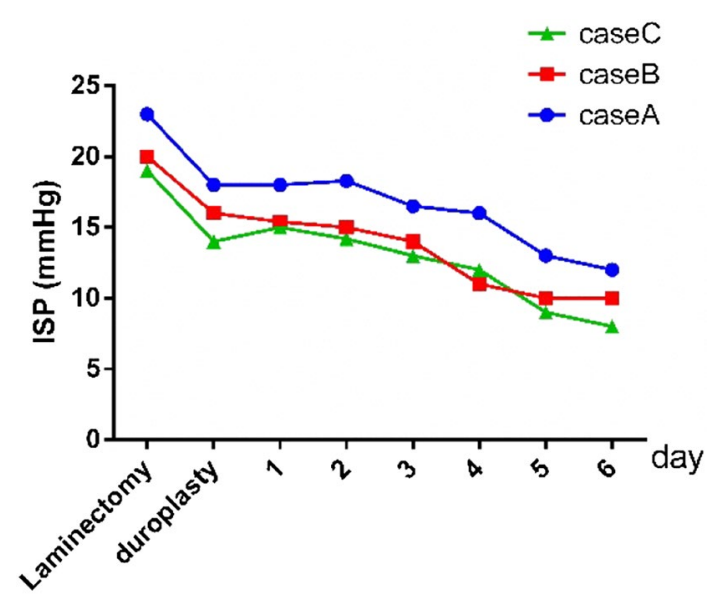

Fig. 3 Results of ISP monitoring in 3 cases. ISP intraspinal pressure

the spinal cord, resulting in sustained compression of the spinal cord. We proposed that spinal cord edema or hemorrhage and contusion, combined with compression of the spinal canal and limitation of the dura, increase the intramedullary and extramedullary pressure and aggravate secondary injury, resulting in spinal cord compartment syndrome similar to osteofascial compartment syndrome $[4,6,13]$.

\section{ISP monitoring}

ISP of three cases was monitored, which showed high ISP even after laminectomy alone, but the ISP decreased steadily after durotomy. The results showed that durotomy significantly reduced ISP. Although there were only 3 cases, we presumed that laminectomy alone was insufficient and that durotomy decompression was needed. Besides, we believe that ISP monitoring helps predict the prognosis of patients. If a high level of ISP is sustained, the spinal cord is also in a state of sustained high pressure, and as the spinal cord is not adequately perfused, the patient may have a worse prognosis. We will conduct ISP monitoring in a large number of cases in the future to explore the relationship between ISP and prognosis.

The surgical method chosen in this study was durotomy after laminectomy. Durotomy is suggested to be performed under a microscope, and this method helps protect the blood vessels on the surface of the spinal cord and avoid iatrogenic injury.

\section{Factors affecting prognosis}

The diagnosis of ASCIWORA is based on MRI findings. Kulkarni summarized several MRI manifestations of this type of SCI: spinal cord edema, spinal cord hemorrhage, spinal cord contusion, and their combination [14]. MRI can predict the prognosis of such patients, and the MRI classification of these diseases has been proposed $[15,16]$. Siddhartha found that for patients with spinal cord edema alone, the results obtained after surgery are often better, while for patients with spinal cord hemorrhage or contusion, the prognosis is poor [17].
Table 6 Results of ISP monitoring in 3 cases

\begin{tabular}{lllllllll}
\hline ISP $(\mathrm{mmHg})$ & Laminectomy & Durotomy & 1 day & 2 day & 3 day & 4 day & 5 day & 6 day \\
\hline Case A & 23 & 18 & 18 & 18.3 & 16.5 & 16 & 13 & 12 \\
Case B & 20 & 16 & 15.4 & 15 & 14 & 11 & 10 & 10 \\
Case C & 19 & 14 & 15 & 14.2 & 13 & 12 & 9 & 8 \\
\hline
\end{tabular}

ISP was monitored every $2 \mathrm{~h}$ after operation. The average daily ISP was taken from the table 
In this study, preoperative MRI findings of SCI were partly confirmed by intraoperative pathology of the spinal cord under intraoperative microscopy. The results showed that the cases of spinal cord hemorrhage or contusion were more severe than those of single spinal cord edema, and the prognosis was relatively poor. However, these cases have recovered to varying degrees, especially in cases of hemorrhage and contusion, indicating that decompression surgery is effective. Figure 4 shows extensive spinal cord edema on MRI and obvious swelling on the surface of the spinal cord and tortuous and swollen blood vessels, indicating that the circulation of the spinal cord is obstructed. After durotomy, the microcirculation of the spinal cord can be improved, and cerebrospinal fluid pulsation was restored. Figure 5 shows MR image of extensive spinal cord edema, localized spinal cord hemorrhage, or contusion on the surface of the spinal cord. Notably, 7 cases of spinal cord hemorrhage or contusion were found on preoperative MRI, while only 4 cases of spinal cord hemorrhage or contusion were confirmed after the durotomy intraoperatively. A possible reason is that some cases of hemorrhage or contusion were not on the surface of the spinal cord. It is thus difficult to identify all cases of spinal cord hemorrhage or contusion under a microscope.

These pathological findings of the spinal cord and its blood vessels suggest that laminectomy alone may not achieve adequate decompression. After opening the dura, the circulation of cerebrospinal fluid can be restored, and the space and environment of the compressed spinal cord and its blood vessels can be optimized. A good environment may help alleviate secondary spinal cord injury and preserve neurological function to the greatest extent. Therefore, herein, we summarized the AMS obtained from their preoperative and final follow-up of the cases of spinal cord injury without fracture and dislocation or central cord syndrome by bony decompression reported in the recent 10 years. In these studies, we found that AMS was lower in our patients before surgery and at the last follow-up this indicated that spinal cord injury was more severe in our patients. However, the growth of AMS in our group was higher than that in most (5/6) groups (Table 7) [18-23].

\section{Complications and its treatment}

Although our surgical method has achieved some curative effect, complications cannot be ignored. Cerebrospinal fluid leakage is the most common complication. Our method of preventing this complication is to suture the dura mater, muscles, and fascia securely. Despite this, 3 cases of cerebrospinal fluid leakage occurred after surgery. As treatment, we used lumbar cistern drainage 10 days after the removal of the cervical drainage tube. Thereafter, no patient developed cerebrospinal fluid infections. All neck wounds healed within 3 weeks. Other complications also had been properly addressed.

\section{Limitations}

This study has some limitations. First, in China, there are a limited number of doctors who perform durotomy for SCI. Second, this study evaluated a relatively small number of cases. Thus, multicenter, prospective studies are needed to prove whether early durotomy is superior to traditional surgical methods [24]. MRI and pathological examination can be used to assess the severity and prognosis of SCI; thus, we may classify these spinal cord injuries by MRI combined with pathology. However, there is still a need to establish a new classification method to evaluate the treatment method and prognosis. Besides, more ISP monitoring cases are thus needed.
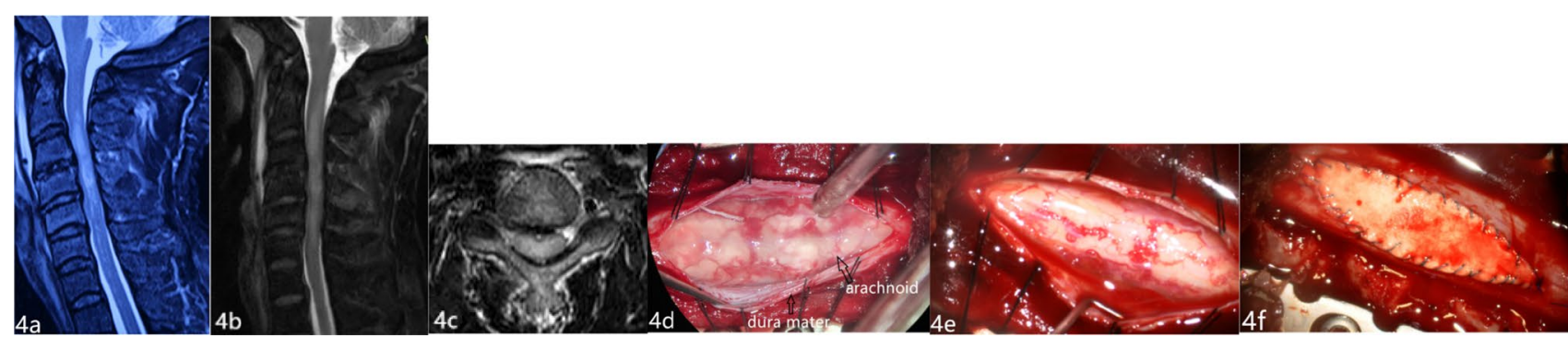

Fig. 4 Durotomy with duroplasty decompression was done within $48 \mathrm{~h}$. a MRI in the local hospital. b, c MRI was done again in our hospital because of decreased blood oxygen saturation. d After incision of the dura mater, the subarachnoid vessels were tortuous and swollen. e The arachnoid was stripped to reach the surface of the spinal cord. The spinal cord was swollen. There were diffuse vascu- lar tortuosity and swelling around the spinal cord with small hemorrhagic foci. f After removing the hematoma, the artificial dura mater was expanded and sutured to provide space for the swelling spinal cord. AIS grade B improved to grade D. AIS American Spinal Injury Association Impairment Scale, MRI magnetic resonance imaging 


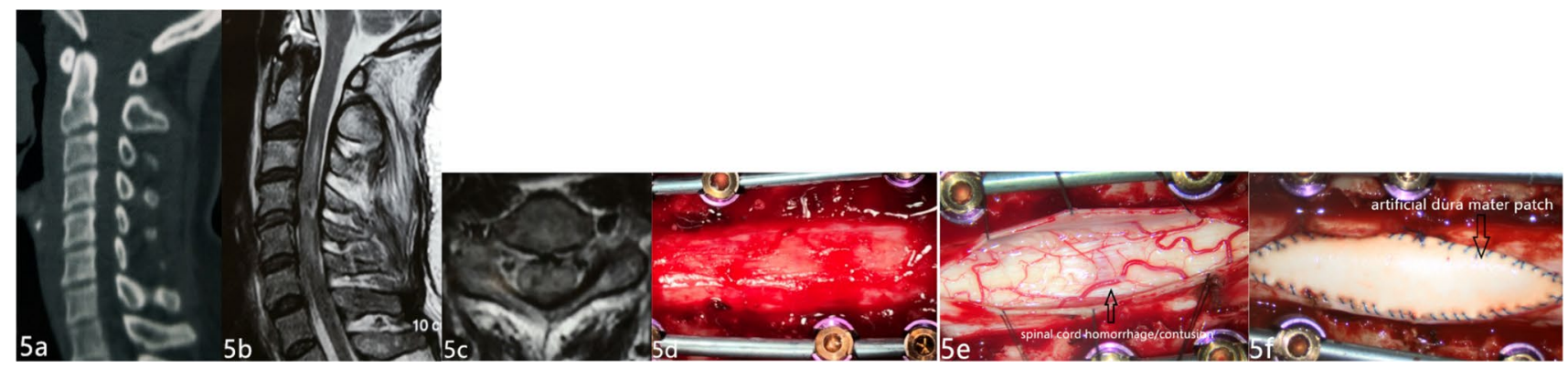

Fig. 5 Durotomy with duroplasty decompression was done within $72 \mathrm{~h}$. a No fracture of the cervical spine was found on CT before operation. b, c MRI showed extensive edema of the spinal cord, similar to hemorrhage or contusion signals in the medulla. d The spinal cord did not regain pulsation after laminectomy. e The arachnoid was cut to reach the surface of the spinal cord. The spinal cord was swollen, and contusion was observed on the surface of the spinal cord. $f$ Duroplasty after thorough decompression. AIS grade B improved to grade D. AIS American Spinal Injury Association Impairment Scale, $C T$ computed tomography, $M R I$ magnetic resonance imaging
Table 7 Evidentiary table of studies evaluating AMS by surgery in spinal cord injury without fracture and dislocation or central cord syndrome

\begin{tabular}{lllll}
\hline References & \multicolumn{2}{l}{ AIS motor score } & & \\
\cline { 2 - 5 } & $\begin{array}{l}\text { Preoperative } \\
\text { AMS }\end{array}$ & $\begin{array}{l}\text { Final follow-up } \\
\text { AMS }\end{array}$ & Description of study $(n)$ & $D$ value \\
\hline This series & 13.1 & 62.6 & Retrospective $(n=16)$ & 49.5 \\
Stevenson et al. [18] & 51 & 96 & Retrospective $(n=22)$ & 46 \\
Anderson et al. [19] & 63.2 & 89.9 & Retrospective $(n=69)$ & 26.7 \\
Aarabi et al. [20] & 63.6 & 94.1 & Retrospective $(n=42)$ & 30.5 \\
Lenehan et al. [21] & 25.2 & 91.3 & Retrospective $(n=70)$ & 66.1 \\
Kawano et al. [22] & 25.1 & 65.1 & Prospective $(n=17)$ & 40 \\
Chen et al. [23] & 54.9 & 89.6 & Retrospective $(n=49)$ & 34.7 \\
\hline
\end{tabular}

\section{Conclusion}

Durotomy helps thoroughly decompress the spinal cord of patients with ASCIWORA and improve the cerebrospinal fluid circulation. Cervical MRI and pathology of SCI can be used to evaluate the severity of damage and the prognosis of those patients to some extent. ISP monitoring is a good method for evaluating intramedullary pressure and decompression.

Funding This study was supported by National Key R\&D Program of China (2016YFC1100100).

\section{Compliance with ethical standards}

Conflict of interest The authors declare that they have no conflict of interest.

Open Access This article is distributed under the terms of the Creative Commons Attribution 4.0 International License (http://creativeco mmons.org/licenses/by/4.0/), which permits unrestricted use, distribution, and reproduction in any medium, provided you give appropriate credit to the original author(s) and the source, provide a link to the Creative Commons license, and indicate if changes were made.

\section{References}

1. Pang D Jr, Wilberger JE (1982) Spinal cord injury without radiographic abnormalities in children. J Neurosurg 69(2):177

2. Kasimatis GB, Panagiotopoulos E, Megas P et al (2008) The adult spinal cord injury without radiographic abnormalities syndrome: magnetic resonance imaging and clinical findings in adults with spinal cord injuries having normal radiographs and computed tomography studies. J Trauma Injury Infect Crit Care 65(1):86-93

3. Thompson C, Gonsalves JF, Welsh D (2015) Hyperextension injury of the cervical spine with central cord syndrome. Eur Spine J 24(1):195-202

4. Qu YZ, Guo XD (2015) Durotomy and dural grafting to treat lower cervical spine injuries with extensive spinal cord edema. Neural Regen Res 10(12):1969-1970

5. Phang I, Werndle MC, Saadoun S et al (2015) Expansion duroplasty improves intraspinal pressure spinal cord, perfusion pressure, and vascular pressure reactivity index in patients with traumatic spinal cord injury: injured spinal cord pressure evaluation study. J Neurotrauma 32(12):865-874

6. Qu Y, Luo Z, Guo X et al (2015) The durotomy or myelotomy for the spinal cord extensive swelling with/without intramedullary hemorrhage. Chin J Orthop 35(007):707-713

7. Layer RT, Ulich TR, Coric D et al (2017) New clinical-pathological classification of intraspinal injury following traumatic acute complete thoracic spinal cord injury: postdurotomy/ myelotomy observations from the INSPIRE trial. Neurosurgery 64(CN_suppl_1):105-109 
8. Aarabi B, Hadley MN et al (2013) Management of acute traumatic central cord syndrome (ATCCS). Neurosurgery 72(2):195-204

9. Allen A (1911) Surgery of experimental lesion of Spinal cord equivalent to crush injury of fracture dislocation of spinal column. JAMA LVII(I 1):878-880

10. Saadoun S, Chen S, Papadopoulos MC (2017) Intraspinal pressure and spinal cord perfusion pressure predict neurological outcome after traumatic spinal cord injury. J Neurol Neurosurg Psychiatry 88(5):452-453

11. Feng YP, Sun TS, Chen L et al (2017) Clinical therapeutic guideline for neurorestoration in spinal cord injury (Chinese version 2016). J Neurorestoratol 5:73-83

12. Grassner L, Grillhösl A, Griessenauer CJ et al (2018) Spinal meninges and their role in spinal cord injury: a neuroanatomical review. J Neurotrauma 35(3):403-410

13. Telemacque D, Zhu F, Guo X et al (2018) Method of decompression by durotomy and duroplasty for cervical spinal cord injury in patients without fracture or dislocation. J Neurorestoratol 6(1):158-164

14. Kulkarni MV, Bondurant FJ (1988) 1.5 tesla magnetic resonance imaging of acute spinal trauma. Radiographics 8(6):1059.

15. Boese CK, Müller D, Bröer R et al (2016) Spinal cord injury without radiographic abnormality (SCIWORA) in adults: MRI type predicts early neurologic outcome. Spinal Cord 54(10):878-883

16. Boese CK, Lechler $P$ (2013) Spinal cord injury without radiologic abnormalities in adults: a systematic review. J Trauma Acute Care Surg 75(2):320-330

17. Siddhartha $S$ (2009) Adult spinal cord injury without radiographic abnormalities (SCIWORA): clinical and radiological correlations. J Clin Med Res 1(3):165-172
18. Stevenson CM, Dargan DP, Warnock J et al (2016) Traumatic central cord syndrome: neurological and functional outcome at 3 years. Spinal Cord 54(11):1010-1015

19. Anderson DG, Sayadipour A, Limthongkul W et al (2012) Traumatic central cord syndrome: neurologic recovery after surgical management. Am J Orthop (Belle Mead, NJ) 41(8):E104-E108

20. Aarabi B, Alexander M, Mirvis SE et al (2011) Predictors of outcome in acute traumatic central cord syndrome due to spinal stenosis. J Neurosurg Spine 14(1):122-130

21. Lenehan B, Fisher CG, Vaccaro A et al (2010) The urgency of surgical decompression in acute central cord injuries with spondylosis and without instability. Spine 35(Supplement):S180-S186

22. Kawano O, Ueta T, Shiba K et al (2010) Outcome of decompression surgery for cervical spinal cord injury without bone and disc injury in patients with spinal cord compression: a multicenter prospective study. Spinal Cord 48(7):548-553

23. Chen L, Yang H, Yang T et al (2009) Effectiveness of surgical treatment for traumatic central cord syndrome. J Neurosurg Spine 10(1):3-8

24. Chen L, Zhang Y, Huang $\mathrm{H}$ et al (2018) Multicenter, randomized, double-blind placebo-control intramedullary decompression for acute complete spinal cord contusion injury. J Neurorestoratol $6(1): 165-170$

Publisher's Note Springer Nature remains neutral with regard to jurisdictional claims in published maps and institutional affiliations.

\section{Affiliations}

\section{Fengzhao Zhu ${ }^{1}$. Sheng Yao ${ }^{1}$. Zhengwei Ren ${ }^{1}$. Dionne Telemacque ${ }^{1} \cdot$ Yanzhen $\mathbf{u}^{1} \cdot$ Kaifang Chen $^{1} \cdot$ Fan Yang $^{1}$. Lian Zeng ${ }^{1} \cdot$ Xiaodong Guo ${ }^{1}$}

Xiaodong Guo

xiaodongguo@hust.edu.cn
1 Department of Orthopaedics, Union Hospital, Tongji Medical College, Huazhong University of Science and Technology, Wuhan 430022, China 